Check for updates

Cite this: RSC Adv., 2019, 9, 19057

\title{
The micro RNA hsa-miR-377-3p inhibits tumor growth in malignant melanoma $\uparrow$
}

\begin{abstract}
Jian Yuan, (D) *a Lei Jiang ${ }^{\mathrm{b}}$ and Chaotang Guo ${ }^{\mathrm{c}}$
Background/Aims: Most recently, micro RNAs (miRNAs/miRs) have been suggested to play a key role in various physiological and pathological processes by regulating the expression of specific genes. The influence of miR-377-3p on multitudinous cancer cells has been investigated; however, its function in melanoma remains undiscovered. Armadillo repeat-containing protein 8 (ARMC8), a target of miR-377$3 p$, plays essential roles in proliferation, differentiation and apoptosis. Our research aimed to detect the specific roles of miR-377-3p in melanoma. Methods: The MiRNA and mRNA expressions were evaluated by a real-time quantitative polymerase chain reaction in the A375 and HEMa-LP cell lines. We predicted the possible interactions between microRNA and mRNAs by bioinformatics database and constructed them with the Cytoscape software. The proliferation and migration activities were investigated using a cell counting kit-8 (CCK8) and wound-healing assay. Validation of the correlation between miR-377-3p and ARMC8 was implemented by the luciferase reporter assay and PCR. Results: The expression of miR377-3p was found to be lower in malignant melanoma cells. The upregulation of miR-377-3p inhibited the melanoma cell proliferation, migration, and ARMC8 expression. miR-377-3p was identified to bind to the $3^{\prime}$ UTR region of ARMC8 directly; this indicated that miR-377-3p suppressed melanoma cell growth partly mediated via the ARMC 8 expression. Conclusion: These findings show that miR-377-3p negatively regulates tumor growth in malignant melanoma, which may thus provide a potential biological target for melanoma treatment and subsequently lead to the development of potential treatments.
\end{abstract}

Received 14th April 2019 Accepted 3rd June 2019 DOI: $10.1039 / c 9 r a 02816 a$ rsc.li/rsc-advances

\section{Introduction}

Malignant melanoma, a cell-type cancer originating from abnormally proliferating melanocytes, is a complex malignancy with significant morbidity and mortality. Malignant melanoma may expand widely throughout the whole body and is the most lethal form of skin cancer. ${ }^{\mathbf{1}}$ The global incidence of malignant melanoma is increasing year after year. ${ }^{2}$ The unfavourable prognosis of malignant melanoma is mainly due to its high migration and lack of effective clinical diagnostic markers. ${ }^{3}$ Thus, there is an urgent need to explore specific disease markers and the underlying molecular mechanisms of melanoma, which may subsequently lead to the development of potential treatments.

MicroRNAs (miRNAs) are a small group of non-coding RNAs that negatively regulate gene expression mainly by binding to the $3^{\prime}$-untranslated region ( $3^{\prime}$ UTR) of specific genes., ${ }^{\mathbf{4} 5}$ MiRNAs

${ }^{a}$ TEDA Institute of Biological Sciences and Biotechnology, Nankai University, Tianjin 300457, P. R. China.E-mail: yj1993@mail.nankai.edu.cn

${ }^{b}$ Department of Medical Technology, Nanyang Medical College, Nanyang, Henan 473000, P. R. China

'Department of Bone, The First People's Hospital of Nanyang, Nanyang, Henan 473000, P. R. China

$\dagger$ Electronic supplementary information (ESI) available. See DOI: 10.1039/c9ra02816a are widely distributed in various tissues among prokaryotes and eukaryotes, ${ }^{6}$ and they play vital regulatory roles in various biological processes including cell proliferation, invasion, apoptosis and tumor initiation, progression metastasis, diagnosis and prognosis. ${ }^{7-9}$ In previous studies, it has been testified that microRNAs contribute to the biological processes of melanoma, ${ }^{7}$ and microRNA profiling has been revealed in melanoma. ${ }^{10}$ MiR-377-3p has been proved to be related to lung cancer. ${ }^{11,12}$ However, melanoma is a complicated process. Therefore, many questions still remain unanswered, and the biological functions of miRNAs in malignant melanoma remain to be further explored.

Armadillo repeat-containing protein 8 (ARMC8), a member of the ARM repeat family, plays essential roles in regulating proliferation, differentiation, apoptosis and so on. ${ }^{\mathbf{1 3 - 1 5}}$ Moreover, most significantly, ARMC8 has been proved to be involved in the prognosis of some other malignancies ${ }^{\mathbf{1 6}}$ and acts as a potential diagnostic marker for certain types of tumors. For example, ARMC8 has been proved to promote the invasiveness and migratory capabilities of colon cancer cells. ${ }^{17}$ In addition, ARMC8 was associated with breast carcinoma, ${ }^{18}$ hepatocellular carcinoma, ${ }^{19}$ lung cancer $^{20}$ and so on. However, its function in malignant melanoma is still largely elusive and needs to be further explored. Consequently, ARMC8 was upregulated in the A375 cell line, a malignant melanoma cell; this indicated that 
ARMC8 enhanced the invasion and metastasis of malignant melanoma.

In our current research, we predicted the relationship between miR-377-3p and melanoma. Subsequently, we have employed a series of biochemical assays to testify the hypothesis that miR-377-3p directly targets ARMC8 mRNA transcripts in melanoma cell, indicating that miR-377-3p is an efficient suppressor of melanoma.

\section{Materials and methods}

\section{Bioinformatics analysis and target prediction}

We detected the predicted miRNAs associated with melanoma via the MNDR v2.0 database ${ }^{21}$ and listed the top 5 putative miRNAs. The target genes of specific miRNA were identified using the miRDB database. We constructed a miRNA-target network using the Cytoscape software 3.5.0 (ref. 22) to visualize their interactions based on our prediction data and the qRT-PCR results for miRNAs and mRNAs.

\section{Cell culture and transfection}

Herein, two human melanocytes-related cell lines, namely the malignant melanoma cell lines A375 and normal epidermal melanocytes HEMa-LP, were obtained from the Shanghai Institute of Cell Biology (Shanghai, China) and cultured in DMEM medium (Solarbio, Beijing, China) with $10 \%$ foetal bovine serum (Hyclone, USA). Cells were incubated in a cell incubator at $37{ }^{\circ} \mathrm{C}$ and $5 \% \mathrm{CO}_{2}$.

Typically, $100 \mathrm{nM}$ miR-377-3p mimics (miR-377-3p), miR377-3p inhibitor (anti-miR-377-3p), miRNA mimic negative controls (miR-NC) obtained from GenePharma (Shanghai, China) were transfected into A375 cells using Lipofectamine 2000 (Invitrogen; Thermo Fisher Scientific, USA), according to the manufacturer's instructions. ARMC8 overexpression plasmid (pcDNA-ARMC8) was constructed via inserting the whole-length sequences of ARMC8 into the pcDNA3.1 vector. A375 cells were grown in 24-well plates and transfected using Lipofectamine 2000 on the next day, with further studies performed at $48 \mathrm{~h}$ post-transfection.

\section{Cell proliferation assay}

The proliferative activity was measured using the cell counting kit-8 (CCK-8 kit) according to the manufacturer's protocol. After transfection, A375 cells were plated into 96-well plates $\left(5 \times 10^{3}\right.$ cells per well), and subsequently, $10 \mu \mathrm{L}$ CCK-8 solution was added to each well for $4 \mathrm{~h}$ and the absorbance at $450 \mathrm{~nm}$ was measured using a fluorescence microplate reader (BioTek).

\section{Cell migration assay}

A total of $5 \times 10^{5}$ A375 cells were cultured in the DMEM medium with $10 \%$ foetal bovine serum in six-well plates placed inside an incubator under $37{ }^{\circ} \mathrm{C}$ and $5 \% \mathrm{CO}_{2}$ conditions overnight. A scratch wound was made with $100 \mu \mathrm{L}$ tips until the cells had grown to $60-80 \%$ confluent. We observed and imaged cell migration inside each culture well using a bright-field inverted microscope (OLYPUS) at 0, 24, 48 and $72 \mathrm{~h}$. Images were further analyzed using ImageJ, ${ }^{23}$ a free software developed by the National Institutes of Health (NIH). This software was used to draw the wounding boundaries of the scratch areas. Therefore, the wound-healing rates indicated the progression of the wounding boundaries. For each experimental condition, three independent runs were performed to obtain the standard error of the mean (SEM).

\section{Quantitative real-time PCR}

Total RNA was extracted using Trizol reagent (Invitrogen, 15596018, USA) from the A375 and HEMa-LP cell lines. RNA quantification and quality were measured by NanoDrop ND-2000 (Thermo, USA).

cDNA synthesis for mciroRNA was performed using miScript II Reverse Transcriptase (Qiagen). The standard procedure was applied for preparation of the cDNA. Quantification of miR-377$3 p$ was performed using the miScript SYBR Green PCR Kit (Qiagen) and detected by a 7500 Fast Real-time PCR System (Applied Biosystems). Relative miRNA levels of each sample were determined by the $\Delta \Delta C_{\mathrm{t}}$ method with small nuclear RNA U6 as a control.

cDNA synthesis for mRNA was performed using the RevertAid First Strand cDNA Synthesis Kit (Thermo Scientific, K1622, USA). The standard procedure for preparation of the cDNA was applied. Quantification of ARMC8 mRNA was performed using a 7500 Fast Real-Time PCR System (Applied Biosystems). The $\Delta \Delta C_{\mathrm{t}}$ method was used to calculate the mRNA relative expression, and the levels of GAPDH were used to normalize the relative expression levels of mRNAs.

The primer sequences for QPCR are shown in Table 1.

\section{Dual-luciferase reporter assay}

The psiCHECK-2 luciferase plasmid carrying wild-type ARMC8 (ARMC8-WT) and mutated ARMC8 (ARMC8-MUT) were purchased from Ke Lei Biological Technology Co., Ltd.,

Table 1 Primer sequences for QPCR

\begin{tabular}{lll}
\hline Gene & Forward primer $\left(5^{\prime}-3^{\prime}\right)$ & Reverse primer $\left(5^{\prime}-3^{\prime}\right)$ \\
\hline GAPDH & GGAGCGAGATCCCTCCAAAAT & GGCTGTTGTCATACTTCTCATGG \\
U6 & CTCGCTTCGGCAGCACA & AACGCTTCACGAATTTGCGT \\
ARMC8 & TGGGAAGTCTTGCTATGGGTA & GTCTGGGGACAGTAGTCCTTG \\
miR-377-3p & ATCACACAAAGGCAACTTTTGT & TGGTGTCGTGGAGTCG
\end{tabular}


Table 2 Top 10 targets of miR-377-3p

\begin{tabular}{|c|c|c|c|c|}
\hline Target rank & Target score & miRNA name & Gene symbol & Gene description \\
\hline 1 & 99 & hsa-miR-377-3p & STOX2 & Storkhead box 2 \\
\hline 2 & 99 & hsa-miR-377-3p & ARMC8 & Armadillo repeat containing 8 \\
\hline 4 & 99 & hsa-miR-377-3p & PUM2 & Pumilio RNA binding family member 2 \\
\hline 5 & 99 & hsa-miR-377-3p & ETS1 & ETS proto-oncogene 1 , transcription factor \\
\hline 6 & 99 & hsa-miR-377-3p & CFTR & $\begin{array}{l}\text { Cystic fibrosis transmembrane conductance } \\
\text { regulator }\end{array}$ \\
\hline 8 & 98 & hsa-miR-377-3p & JAG1 & Jagged 1 \\
\hline 9 & 98 & hsa-miR-377-3p & SETBP1 & SET binding protein 1 \\
\hline 10 & 98 & hsa-miR-377-3p & DCAF12 & DDB1 and CUL4 associated factor 12 \\
\hline
\end{tabular}

(Shanghai, China). Subsequently, cells were cotransfected with luciferase reporter plasmid transfected with miR-377-3p mimics or miR-NC using lipofectamine 2000. Luciferase activities were measured using a microplate reader (Bio Tek) after $48 \mathrm{~h}$.

\section{Western blot assay}

The proteins for the Western blot analysis were obtained from cells lysed using RIPA lysis buffer (Wanleibio, Shenyang, China) containing 1\% PMSF. Total protein extracts were separated by $10 \%$ SDS-PAGE under the standard routine, transferred to PVDF membranes, using anti-ARMC8 antibody (ab108158; $1: 2000$ dilution; Abcam; Cambridge, England) and HRP-conjugated goat anti-rabbit secondary antibody (WLA023a; diluted at $1: 600$; Wanleibio) to detect ARMC8 protein expression. The blots were visualized with ECL Plus Western blotting detection reagent (WLA006; Wanleibio).

\section{In vivo tumorigenicity assay}

The 8 week old C57BL/6 mice were obtained from the SPF (Beijing, China) Biotechnology Co., Ltd. All animal studies were

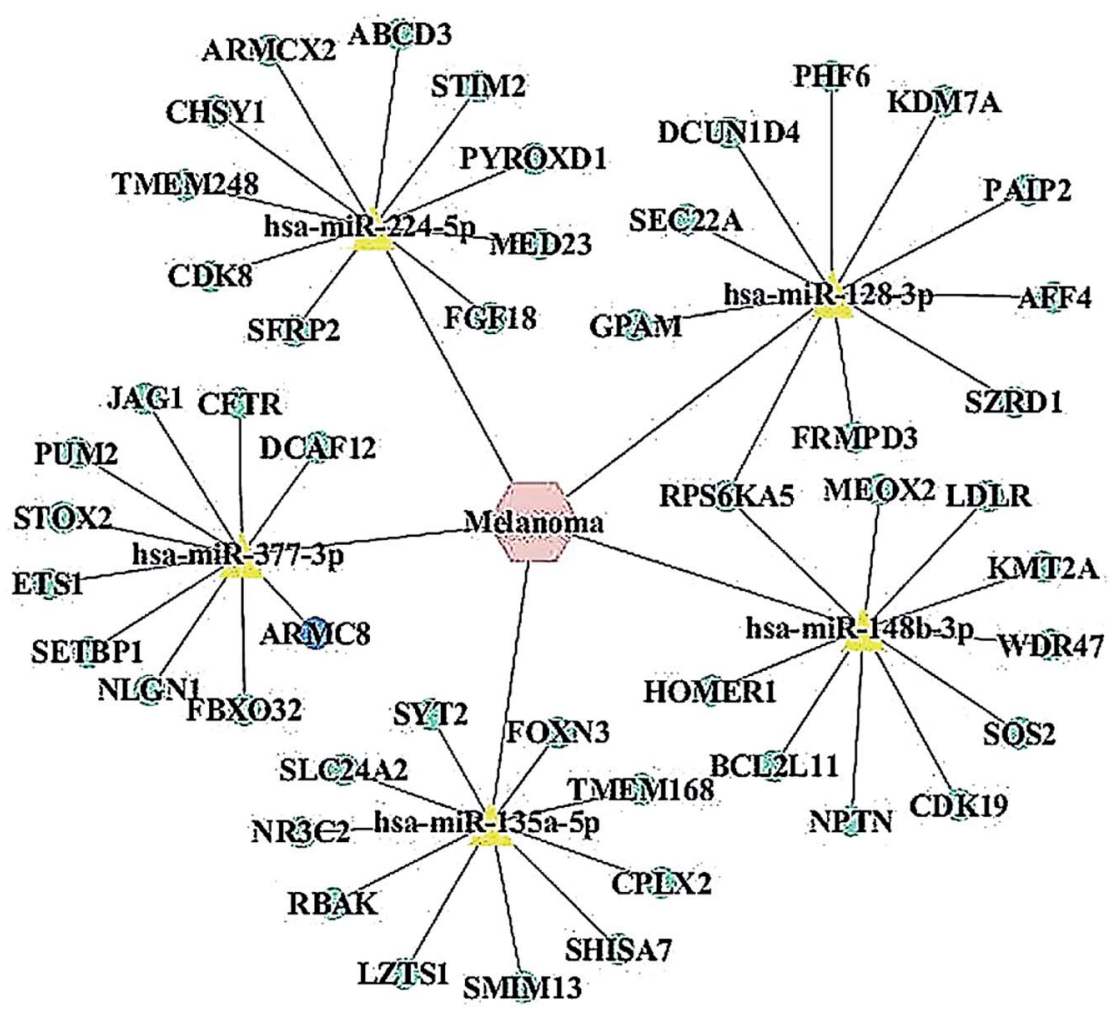

Fig. 1 Specific network of miRNA-mRNAs associated with melanoma. We chose the top 5 miRNAs and the top 10 predicted target genes of microRNAs according to a target score $>90$ in the miRDB database. We used Cytoscape software 3.5.0 to visualize miRNA-target genes interactions based on the miRNAs prediction and miRNA target genes prediction results. In the network, 5 miRNAs were related to melanoma and 50 target genes were related to the melanoma of miRNA. The yellow triangles represent miRNAs; the green circles represent the target genes of miRNAs and the blue circles represent ARMC8. The relationships between the nodes are connected by the solid lines. 
performed according to the animal use guidelines of the National Institute of Health and the current Chinese regulations and standards on the use of laboratory animals. A total of $5 \times 10^{5}$ cells were injected subcutaneously into mice and the tumour volume was measured at 5, 10, 15, 20, 25 and $30 \mathrm{~d}$. The mice were sacrificed at $30 \mathrm{~d}$ after injection. The tumour volume was evaluated using the following formula: tumour volume $=$ $4 \pi / 3 \times(\text { width } / 2)^{2} \times($ length $/ 2)$. All the mice had free access to food and water and were raised on a $12 \mathrm{~h}$ light/12 h dark cycle at $20{ }^{\circ} \mathrm{C}$. All animal experiments were performed in accordance with the Guidelines for Care and Use of Laboratory Animal and were approved by the Animal Research Committee of Nankai University.

\section{Statistical analysis}

SPSS 21.0 version and Graph pad prism 7.0 were used for the statistical analyses. Data are presented as the mean \pm standard deviations (SDs) for triplicate measurements. Student's independent $t$ test was used to compare statistical differences between different groups and a $p$-value $<0.05$ was considered statistically significant.

\section{Results}

\section{Prediction and construction of the miRNA-target interaction network}

Recent evidences have demonstrated that miRNAs play a crucial role in the regulation of gene expression by sequestering the
mRNAs. ${ }^{24}$ We detected the predicted miRNAs associated with melanoma via the MNDR v2.0 database and listed the top 5 putative miRNAs as miR-148b-3p, miR-377-3p, miR-224-5p, miR-128-3p and miR-135a-5p. All the putative miRNAs are listed in ESI Table 1. $\dagger$ We found out the miRNA targets using the miRDB database and listed the top 10 putative mRNAs for miR377-3p (Table 2). All the putative mRNAs for miR-377-3p are listed in the ESI Table $2 . \dagger$ The miRNA-target network was constructed to visualize their interactions based on the miRNA target prediction results via the Cytoscape 3.5.0 software (Fig. 1). Moreover, one cancer-related gene, ARMC8, which was a potential target of miR-377-3p, was listed up with high scores.

\section{miR-377-3p was downregulated in A375 cells}

To validate the level of miR-377-3p in malignant melanoma, the miRNA qRT-PCR assay was further performed using the A375 and HEMa-LP) cell lines. As expected, we found that the relative expression of miR-377-3p in A375 cells was downregulated over three times that in the HEMa-LP cells (Fig. 2a). To validate the level of miR-377-3p in malignant melanoma, the miRNA qRTPCR assay was further performed using the A375 and HEMaLP cell lines. As expected, we found that the expression of miR-377-3p in A375 cells was downregulated over three times that in the HEMa-LP cells (Fig. 2a). Thus, miR-377-3p inhibited the proliferation and migration of the A375 cells.

To further investigate the function of miR-377-3p in melanoma cells, the A375 cells were transfected with miR-377-3p mimics or miR-NC. The QRT-PCR results showed that the
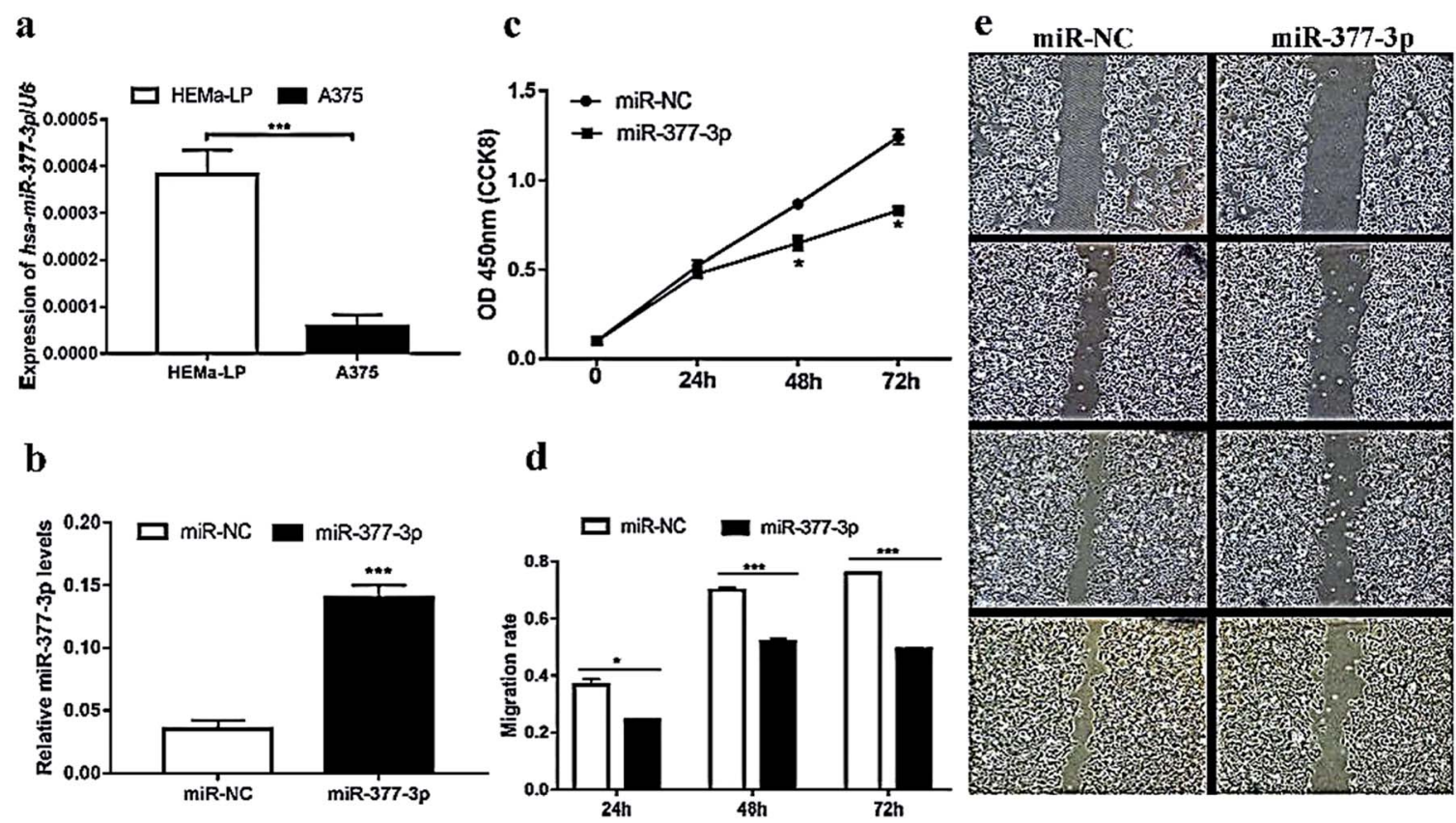

Fig. 2 miR-377-3p suppressed the proliferation and migration of melanoma cells. (a) miR-377-3p was downregulated in the A375 cell line and the $\Delta \Delta C_{t}$ method was used to calculate the relative expression with small nuclear RNA U6 as a control $(* * * p<0.001)$. (b) miR-377-3p level was significantly improved after miR-377-3p mimics were treated in A375 cells (***p $<0.001$ ). (c) A CCK-8 experiment was used to detect cell proliferation after treatment with miR-NC or miR-377-3p mimics in A375 cells (*p<0.05). ( $d$ and e) A straight scratch wound assay was used to compare the migration rate of melanoma cells between different groups, cell migration was inhibited after miR-377-3p mimics treatment $(* p<$ $0.05, * * * p<0.001$ ). 
expression of miR-377-3p was induced by the miR-377-3p mimics (Fig. 2b). The CCK-8 assay suggested that the upregulation of miR-377-3p significantly decreased cell proliferation (Fig. 2c) in the A375 cell line. A scratch wound assay was used to determine the migration rate of melanoma cells, and it was found that cell migration decreased when the A375 cells were transfected with the miR-377-3p mimics (Fig. 2d and e); this indicated that miR-377-3p inhibited melanoma cell migration.

\section{miR-377-3p directly binds to ARMC8 to suppress tumor cell growth}

For further exploration, we next detected the ARMC8 mRNA level in malignant melanoma. As expected, ARMC8 was upregulated in the A375 cells (Fig. 3a); this suggested that ARMC8 promoted melanoma progression. In addition, the ARMC8 expression was inhibited after miR-377-3p was transfected (Fig. 3b); this indicated that ARMC8 might be mediated by miR377-3p. We identified the binding sites between miR-377-3p and ARMC8 using RNA22 version 2.0 (ref. 25) and further illustrated the binding site (Fig. 3c). The luciferase reporter assay shows that the luciferase activity of the reporter plasmid containing wild-type ARMC8 was markedly lowered in the A375 cells with miR-377-3p overexpression; however, no obvious change was observed in the mutated ARMC8 reporter (Fig. 3d). The Western blotting results manifested that the ARMC8 protein level was dramatically inhibited by miR-377-3p in the A375 cell line (Fig. 3e). Overall, miR-377-3p suppresses melanoma via directly binding to $3^{\prime}$ UTR of ARMC8 in the A375 cell line.

\section{miR-377-3p inhibits melanoma tumor formation in vivo}

Furthermore, we investigated the effects of miR-377-3p on tumour growth in vivo. The A375 cells were transfected with NC or miR-377-3p. The transfected cells $\left(5 \times 10^{5}\right.$ cells $)$ were injected into the mice. The dissected tumors were imaged and are shown in Fig. 4a, and the tumor volume obtained after injection is shown in Fig. 4b. The results showed that miR-377-3p decreased the tumour volume. Moreover, we extracted total RNA from the dissected tumors and detected the expression of miR-377-3p and ARMC8 (Fig. 4c and d). The results obtained from the qRT-PCR assay showed that the overexpression of miR-377-3p inhibited the ARMC8 mRNA expression in the mice model, $\mathbf{a}$

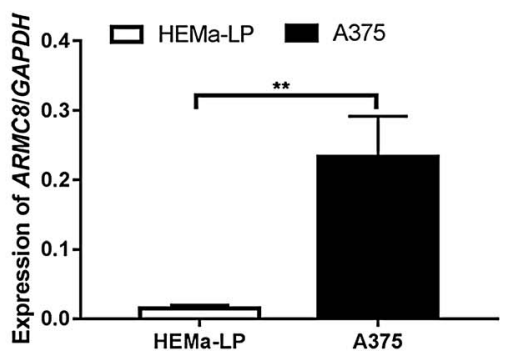

b

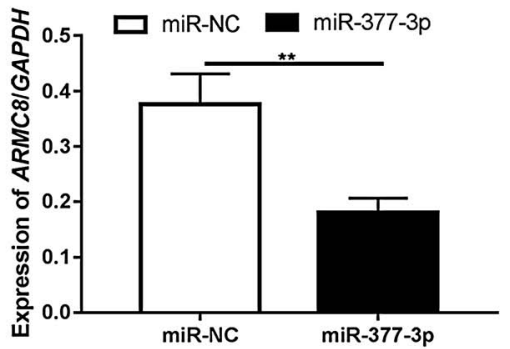

c hsa-miR-377-3p 3'-UGUUUUCAACGGAAACACACUA-5'

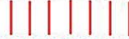

ARMC8-3'UTR 5'-AUGAAUGGAUGUGUGUGUGUGAG-3'

(WT)

ARMC8-3'UTR 5'-AUGAAUGGAUGUGUGCAGUGUGG-3'

(MUT)

d

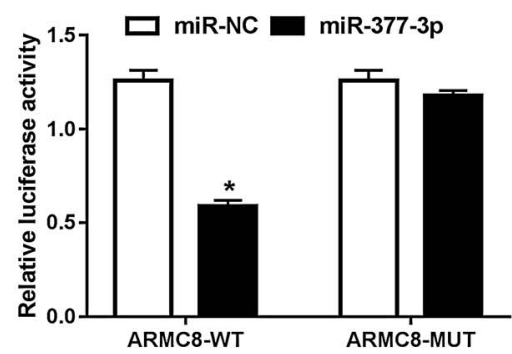

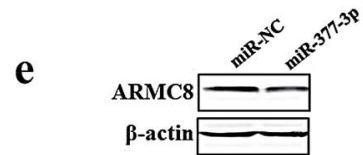



Fig. 3 miR-377-3p inhibited the ARMC8 mRNA level through binding to its 3 '-untranslated region directly. (a) ARMC8 mRNA was upregulated in the human malignant melanoma A375 cell line and detected by qRT-PCR using the $\Delta \Delta C_{\mathrm{t}}$ method with GAPDH as a control (** $p<0.01$ ). (b) ARMC8 mRNA expression was notably decreased following the treatment of miR-377-3p mimics in A375 cells (**p<0.01). (c) The putative binding sites between the ARMC $83^{\prime}$-untranslated region and miR-377-3p. (d) Luciferase activity of the reporter containing wild-type or mutated ARMC 8 genes was assessed in A375 cells transfected with miR-NC or miR-377-3p. (e) The relative protein level of ARMC 8 was measured by Western blot after the transfection of miR-377-3p mimics or miR-NC in A375 cells with $\beta$-actin as a control $(* p<0.05)$. 
a

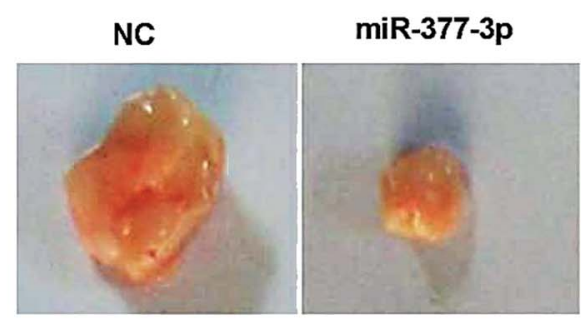

C

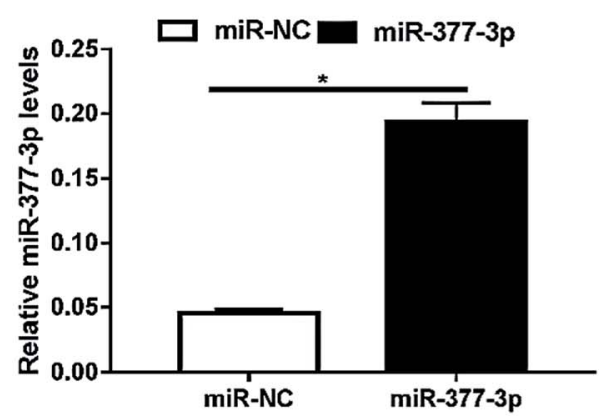

b

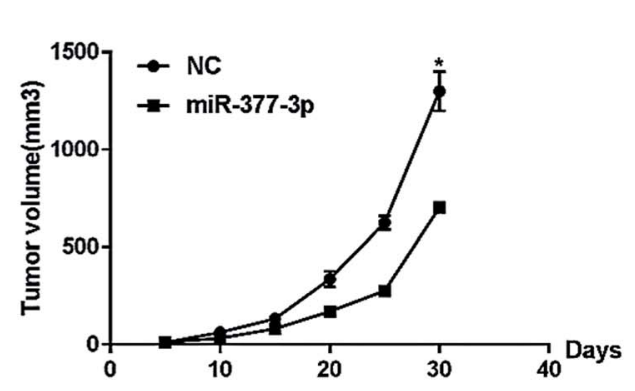

d

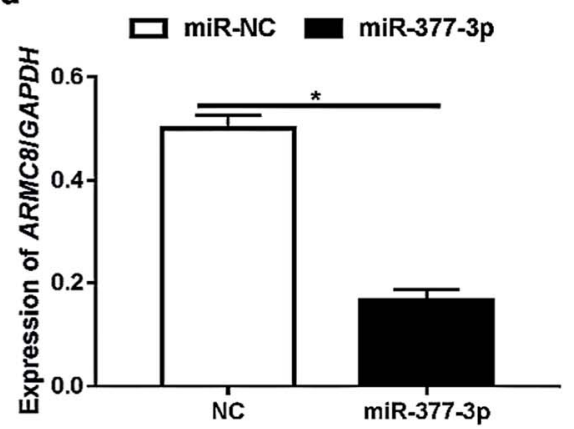

Fig. 4 miR-377-3p suppresses melanoma cell growth in vivo. The A375 cells overexpressing miR-377-3p were injected subcutaneously into mice; (a) the mice were sacrificed at 30 days after injection, and the tumors were isolated; (b) the tumor volume was calculated $(* p<0.05)$; (c) the miR-377-3p level in the tumor tissue was measured using real-time PCR ( $*<<0.05)$; and (d) the ARMC 8 mRNA level in the tumor tissue was detected using real-time PCR $(* p<0.05)$.

indicating that miR-377-3p inhibited melanoma growth by inhibiting the ARMC8 expression.

\section{Discussion}

Recent studies have demonstrated that miRNAs can function as mRNA suppressors during the regulation of genes, such as oncogene, in many diseases and biological processes, indicating that miRNAs may serve as tumor suppressors. ${ }^{26}$ The miRNA profiling for clinical skin diseases, such as squamous cell carcinoma, equine sarcoids, and cutaneous melanoma, has been reported. ${ }^{27-29}$ Moreover, researchers are trying to discover, exploit and identify miRNAs that may serve as either diagnostic markers or therapeutic targets for numerous different kinds of tumors. ${ }^{30,31}$ MiRNAs also participate in tumor metastasis, growth, proliferation, migration and drug resistance in melanoma. ${ }^{32-34}$ In addition, evidence has showed that miRNA-193b suppresses cell proliferation by directly binding to cyclin D1 in the melanoma cell line Malme-3M. ${ }^{35}$ During the transformation of melanocytes, the miRNA profiles exhibit a striking change, and upregulated miRNAs as a melanoma tumor suppressor may inhibit the mRNA expression to influence cell proliferation and differentiation..$^{36}$ Therefore, the important roles of miRNAs in skin melanoma function regulation, including those in proliferation, differentiation, migration and carcinogenesis, have slowly started to attract more attention.

MiR-377-3p, mentioned as a tumor suppressor in many literature studies, negatively regulates glioma cell proliferation and migration ${ }^{37}$ and is a prognostic marker in squamous cell carcinoma. ${ }^{38}$ Moreover, miR-377-3p is also involved in some stem cell differentiation. ${ }^{39}$ The specific functions of miR-377-3p in a great deal of human cancers have been identified; however, limited data are available about its changes and roles in cutaneous malignant melanoma. Thus, exploration of its function in malignant melanoma cells might provide us with some new hints from some untouched aspects. In this study, we performed micro RNA quantification to validate the expression level of miR-377-3p. Consistent with our expectation, miR-377$3 p$ was identified to be downregulated in malignant melanoma cells. Thus, miR-377-3p might play vital roles in the regulation of melanoma progression, and the mechanisms need to be further explored.

MiRNAs regulate gene expression in both the mRNA translation phase and the post-transcriptional modification level. In this study, we performed predictions for the miRNA/mRNA interaction network to explore the potential function mechanisms, especially related to the melanoma biological function. Herein, our network prediction indicated that ARMC8, an essential member of the armadillo repeat family, might serve as a potential target for miR-377-3p, which was significantly reduced in malignant melanoma cells. ARMC8 was proved to promote proliferation and inhibit apoptosis in ovarian cancer and osteosarcoma cells. ${ }^{13,14}$ ARMC8 also regulates the invasive ability of hepatocellular carcinoma. ${ }^{19}$ Based on the abovementioned findings and our results, we speculate that miR-3773p may suppress the ARMC8 mRNA expression, which in turn inhibits melanoma tumor cell growth. 
As is well-known, the interaction between miRNAs and their mRNA targets is complicated. The mechanisms of action of microRNA are still controversial. Researchers have proposed several models to explain its mechanisms, ${ }^{\mathbf{4 0 , 4 1}}$ but its mechanisms need to be further explored.

Regarding the limitations of this research, we have only provided circumstantial evidence for the possible biological functional way via which miR-377-3p regulates melanoma biological function including proliferation, migration, and invasion. Further exploration to directly verify the association between micro RNA and mRNA and their underlying mechanisms in regulating the melanoma function is required and will be performed in our future study.

In conclusion, our studies revealed that miR-377-3p suppressed tumor growth in malignant melanoma, indicating that miR-377-3p might be a potential therapeutic target for melanoma patients. We also provided theoretic insights into the molecular mechanisms of miR-377-3p as a potential therapeutic target of some related diseases.

\section{Conflicts of interest}

There are no conflicts to declare.

\section{Acknowledgements}

This research did not receive any specific grant from funding agencies in the public, commercial, or not-for-profit sectors. This research was supported by the TEDA Institute of Biological Sciences of Nankai University. We thank all members in this department for their assistance. We thank all author's contributions who participated in this study. We thank all members from department of Medical Technology of Nanyang Medical College for their convenience for our experiments. The authors would like to thank Weisong Wang (University of Jinan) for his help with data analysis.

\section{References}

1 A. Millet, A. R. Martin, C. Ronco, S. Rocchi and R. Benhida, Metastatic Melanoma: Insights Into the Evolution of the Treatments and Future Challenges, Med. Res. Rev., 2017, 37, 98-148.

2 E. G. Little and M. J. Eide, Update on the current state of melanoma incidence, Dermatol. Clin., 2012, 30, 355-361.

3 A. E. Russo, F. Ferrau, G. Antonelli, D. Priolo, J. A. McCubrey and M. Libra, Malignant melanoma in elderly patients: biological, surgical and medical issues, Expert Rev. Anticancer Ther., 2015, 15, 101-108.

4 B. P. Lewis, C. B. Burge and D. P. Bartel, Conserved seed pairing, often flanked by adenosines, indicates that thousands of human genes are microRNA targets, Cell, 2005, 120, 15-20.

5 B. Zhan, X. Pan, G. P. Cobb and T. A. Anderson, MicroRNAs as oncogenes and tumor suppressors, Dev. Biol., 2007, 302, $1-12$.
6 G. A. Calin and C. M. Croce, MicroRNA signatures in human cancers, Nat. Rev. Cancer, 2006, 6, 857-866.

7 W. Luan, Y. Qian, X. Ni, X. Bu, Y. Xia, J. Wang, H. Ruan, S. Ma and B. Xu, miR-204-5p acts as a tumor suppressor by targeting matrix metalloproteinases-9 and B-cell lymphoma-2 in malignant melanoma, OncoTargets Ther., 2017, 10, 1237-1246.

8 C. Jiang, X. Hu, M. Alattar and H. Zhao, miRNA expression profiles associated with diagnosis and prognosis in lung cancer, Expert Rev. Anticancer Ther., 2014, 14, 453-461.

9 J. Xiong, Q. Du and Z. Liang, Tumor-suppressive microRNA22 inhibits the transcription of E-box-containing c-Myc target genes by silencing c-Myc binding protein, Oncogene, 2010, 29, 4980-4988.

10 M. Aksenenko, N. Palkina, A. Komina, L. Tashireva and T. Ruksha, Differences in microRNA expression between melanoma and healthy adjacent skin, BMC Dermatol., 2019, 19, 1.

11 C. Sun, S. Li, F. Zhang, Y. Xi, L. Wang, Y. Bi and D. Li, Long non-coding RNA NEAT1 promotes non-small cell lung cancer progression through regulation of miR-377-3p-E2F3 pathway, Oncotarget, 2016, 7, 51784-51814.

12 J. Zhang, Y. Li, M. Dong and D. Wu, Long non-coding RNA NEAT1 regulates E2F3 expression by competitively binding to miR-377 in non-small cell lung cancer, Oncol. Lett., 2017, 14, 4983-4988.

13 G. Jiang, D. Yang, L. Wang, X. Zhang, H. Xu, Y. Miao, E. Wang and Y. Zhang, A novel biomarker ARMc8 promotes the malignant progression of ovarian cancer, Hum. Pathol., 2015, 46, 1471-1479.

14 F. Jiang, Y. Shi, H. Lu and G. Li, Armadillo RepeatContaining Protein 8 (ARMC8) Silencing Inhibits Proliferation and Invasion in Osteosarcoma Cells, Oncol. Res., 2016, 24, 381-389.

15 X. Liang, Q. L. Men, Y. W. Li, H. C. Li, T. Chong and Z. L. Li, Silencing of Armadillo Repeat-Containing Protein 8 (ARMc8) Inhibits TGF-beta-Induced EMT in Bladder Carcinoma UMUC3 Cells, Oncol. Res., 2017, 25, 99-105.

16 D. Zhou, W. Zhang, Y. Wang, L. Chen and J. Luan, ARMc8: a potential diagnostic and therapeutic target for cancers, Hum. Pathol., 2016, 54, 201.

17 G. Jiang, Y. Zhang, X. Zhang, C. Fan, L. Wang, H. Xu, J. Yu and E. Wang, ARMc8 indicates aggressive colon cancers and promotes invasiveness and migration of colon cancer cells, Tumor Biol., 2015, 36, 9005-9013.

18 C. Fan, Y. Zhao, X. Mao, Y. Miao, X. Lin, G. Jiang, X. Zhang, Q. Han, L. Luan and E. Wang, Armc8 expression was elevated during atypia-to-carcinoma progression and associated with cancer development of breast carcinoma, Tumor Biol., 2014, 35, 11337-11343.

19 Y. Zhao, S. Peng, C. Jia, F. Xu, Y. Xu and C. Dai, Armc8 regulates the invasive ability of hepatocellular carcinoma through E-cadherin/catenin complex, Tumor Biol., 2016, 37, 11219-11224.

20 C. Xie, G. Jiang, C. Fan, X. Zhang, Y. Zhang, Y. Miao, X. Lin, J. Wu, L. Wang, Y. Liu, J. Yu, L. Yang, D. Zhang, K. Xu and E. Wang, ARMC8alpha promotes proliferation and 
invasion of non-small cell lung cancer cells by activating the canonical Wnt signaling pathway, Tumor Biol., 2014, 35, 8903-8911.

21 T. Cu, L. Zhang, Y. Huang, Y. Yi, P. Tan, Y. Zhao, Y. Hu, L. Xu, E. Li and D. Wang, MNDR v2.0: an updated resource of ncRNA-disease associations in mammals, Nucleic Acids Res., 2018, 46, D371-D374.

22 M. Kohl, S. Wiese and B. Warscheid, Cytoscape: software for visualization and analysis of biological networks, Methods Mol. Biol., 2011, 696, 291-303.

23 C. Venter and C. U. Niesler, Rapid quantification of cellular proliferation and migration using ImageJ, BioTechniques, 2019, 66, 99-102.

$24 \mathrm{H}$. Qin and W. Liu, MicroRNA-99a-5p suppresses breast cancer progression and cell-cycle pathway through downregulating CDC25A, J. Cell. Physiol., 2019, 234, 35263537.

25 K. C. Miranda, T. Huynh, Y. Tay, Y. S. Ang, W. L. Tam, A. M. Thomson, B. Lim and I. Rigoutsos, A pattern-based method for the identification of MicroRNA binding sites and their corresponding heteroduplexes, Cell, 2006, 126, 1203-1217.

26 I. Koturbash, F. J. Zemp, I. Pogribny and O. Kovalchuk, Small molecules with big effects: the role of the microRNAome in cancer and carcinogenesis, Mutat. Res., 2011, 722, 94-105.

27 O. Stojadinovic, H. Ramirez, I. Pastar, K. A. Gordon, R. Stone, S. Choudhary, E. Badiavas, K. Nouri and M. Tomic-Canic, MiR-21 and miR-205 are induced in invasive cutaneous squamous cell carcinomas, Arch. Dermatol. Res., 2017, 309, 133-139.

28 K. Pawlina, A. Gurgul, T. Szmatoła, C. Koch, K. Mählmann, M. Witkowski and M. Bugno-Poniewierska, Comprehensive characteristics of microRNA expression profile of equine sarcoids, Biochimie, 2017, 137, 20-28.

29 V. Armand-Labit, N. Meyer, A. Casanova, H. Bonnabau, V. Platzer, E. Tournier, B. Sansas, S. Verdun, B. Thouvenot, B. Hilselberger, A. Doncescu, L. Lamant, M. Lacroix-Triki, G. Favre and A. Pradines, Identification of a Circulating MicroRNA Profile as a Biomarker of Metastatic Cutaneous Melanoma, Acta Derm.-Venereol., 2016, 96, 29-34.

30 C. Rovira, M. C. Guida and A. Cayota, MicroRNAs and other small silencing RNAs in cancer, IUBMB Life, 2010, 62, 859868 .
31 S. P. Nana-Sinkam and C. M. Croce, MicroRNAs as therapeutic targets in cancer, Transl. Res., 2011, 157, 216225.

32 J. Noori, M. Sharifi and S. Haghjooy Javanmard, miR-30a Inhibits Melanoma Tumor Metastasis by Targeting the Ecadherin and Zinc Finger E-box Binding Homeobox 2, Adv. Biomed. Res., 2018, 7, 143.

33 M. Kunz, MicroRNAs in melanoma biology, Adv. Exp. Med. Biol., 2013, 774, 103-120.

34 S. Liu, M. T. Tetzlaff, R. Cui and X. Xu, miR-200c inhibits melanoma progression and drug resistance through downregulation of BMI-1, Am. J. Pathol., 2012, 181, 1823-1835.

35 J. Chen, H. E. Feilotter, G. C. Paré, X. Zhang, J. G. Pemberton, C. Garady, D. Lai, X. Yang and V. A. Tron, MicroRNA-193b represses cell proliferation and regulates cyclin D1 in melanoma, Am. J. Pathol., 2010, 176, 2520-2529.

36 C. R. Gasque Schoof, A. Izzotti, M. G. Jasiulionis and R. Vasques Ldos, The Roles of miR-26, miR-29, and miR203 in the Silencing of the Epigenetic Machinery during Melanocyte Transformation, Biomed. Res. Int., 2015, 2015, 634749.

37 Y. Liu, Y. Gao, D. Li, L. He, L. Iw, B. Hao, X. Chen and Y. Cao, LASP1 promotes glioma cell proliferation and migration and is negatively regulated by miR-377-3p, Biomed. Pharmacother., 2018, 108, 845-851.

38 M. E. 1. Baroudi, J. P. Machiels and S. Schmitz, Expression of SESN1, UHRF1BP1, and miR-377-3p as prognostic markers in mutated TP53 squamous cell carcinoma of the head and neck, Cancer Biol. Ther., 2017, 18, 775-782.

39 X. Li, Y. Yang, R. Yan, X. Xu, L. Gao, J. Mei, J. Liu, X. Wang, J. Zhang, P. Wu, W. Li, Z. Zhao, J. Xiong and T. Wang, miR377-3p regulates adipogenic differentiation of human bone marrow mesenchymal stem cells by regulating LIFR, Mol. Cell. Biochem., 2018, 449, 295-303.

40 W. Filipowicz, S. N. Bhattacharyya and N. Sonenberg, Mechanisms of post-transcriptional regulation by microRNAs: are the answers in sight?, Nat. Rev. Genet, 2008, 9, 102-114.

41 S. Oliveto, M. Mancino, N. Manfrini and S. Biffo, Role of microRNAs in translation regulation and cancer, World $J$. Biol. Chem., 2017, 8, 45-56. 